\title{
Protein kinase CK2 mediates peroxynitrite-induced heme oxygenase-1 expression in articular chondrocytes
}

\author{
KANG MI KIM ${ }^{1}$, JU DONG SONG ${ }^{1}$, HUN TAEG CHUNG ${ }^{2}$ and YOUNG CHUL PARK ${ }^{1}$ \\ ${ }^{1}$ Department of Microbiology and Immunology, Pusan National University School of Medicine, Yangsan, \\ Gyeongnam 626-870; ${ }^{2}$ School of Biological Sciences, Ulsan University, Ulsan 680-749, Republic of Korea
}

Received January 9, 2012; Accepted January 30, 2012

DOI: $10.3892 / \mathrm{ijmm} .2012 .949$

\begin{abstract}
Heme oxygenase-1 (HO-1) is induced as an adaptive mechanism against oxidative stress in chondrocytes, which play an important role in the maintenance and degradation of cartilage. In the present study, we examined the role of protein kinase casein kinase (CK2) on peroxynitrite-induced expression of HO-1 in primary articular chondrocytes. 3-Morpholinosydnonimine hydrochloride (SIN-1) has been shown to mediate cell death by activating apoptosis-related molecules in cells. In this study, we used a low concentration of SIN-1 that did not induce apoptosis to elucidate the mechanism by which SIN-1 upregulates HO-1 expression. In chondrocytes, SIN-1 induced HO-1 expression with spontaneous downregulation in a different manner than with high concentrations of SIN-1. Importantly, SIN-1 treatment of chondrocytes increased CK2 activation. Additionally, inhibition of CK2 with 4,5,6,7-tetrabromobenzotriazole (TBB) or siRNA did not induce HO-1 expression and reduced NF-E2-related factor 2 (Nrf2) accumulation in chondrocytes. Therefore, we examined whether CK2 directly regulates $\mathrm{Nrf2}$, which is a transcription factor that regulates the expression of HO-1. Indeed, TBB treatment inhibited phosphorylation and nuclear translocation of Nrf2 in SIN-1-treated cells. Moreover, using an immunoprecipitation assay, we confirmed that SIN-1 treatment enhanced the interaction between CK2 and Nrf2. Taken together, our findings suggest that peroxynitrite activates Nrf2
\end{abstract}

Correspondence to: Dr Young Chul Park, Department of Microbiology and Immunology, Pusan National University School of Medicine, Yangsan, Gyeongnam 626-870, Republic of Korea E-mail: ycpark@pusan.ac.kr

Abbreviations: HO-1, heme oxygenase-1; protein kinase CK2, protein kinase casein kinase 2; SIN-1, 3-morpholinosydnonimine hydrochloride; NO, nitric oxide; Nrf2, NF-E2-related factor 2; ROS, reactive oxygen species; DRB, 5,6-dichlorobenzimidazole riboside; PI, propidium iodide; TUNEL, terminal deoxynucleotidyl transferase dUTP nick end labeling; HRP, horseradish peroxidase; ECL, enhanced chemiluminescence

Key words: HO-1 expression, peroxynitrite, protein kinase CK2, Nrf2, chondrocytes via CK2 signaling, leading to the upregulation of HO-1 in primary chondrocytes.

\section{Introduction}

Heme oxygenase-1 (HO-1) catabolizes heme to form carbon monoxide, free iron, and biliverdin (1). These metabolic products play important roles in both physiology and pathology $(2,3)$. In general, $\mathrm{HO}-1$ is thought to protect cells and tissues from oxidative stress-induced damage $(2,4)$. HO-1 is also anti-inflammatory, antiproliferative, and antiapoptotic through modulating gene expression and enzymatic activities (5-7). Recent reports suggest that the induction of HO-1 results in the downregulation of catabolic enzymes and inflammatory mediators in chondrocytes; this in turn suggests a role for HO-1 in the defense systems of articular cartilage $(8,9)$.

HO-1 is strongly induced by a variety of stimuli such as inflammatory cytokines, ultraviolet radiation, heme, and heavy metals. The HO-1 promoter contains several transcriptional regulatory elements that respond to redox-sensitive transcription factors. NF-E2-related factor 2 (Nrf2) is the main factor regulating transcriptional activation of HO-1 (10). Nrf2's activity is tightly regulated by its interaction with the actin-binding protein Keap1, a negative modulator of Nrf2 (11). Nrf2 phosphorylation leads to dissociation with Keap1 in the cytosol and nuclear localization of Nrf2. It has reported that phosphatidylinositol 3-kinase (PI3K) and extracellular signal-regulated kinase (ERK) regulate Nrf2 activation in stress-induced cells $(12,13)$.

Protein kinase CK2, formerly called casein kinase 2, is a ubiquitous and highly conserved serine/threonine protein kinase consisting of 2 catalytic subunits $\left(\alpha \alpha, \alpha \alpha^{\prime}, \alpha^{\prime} \alpha^{\prime}\right)$ and 2 regulatory $\beta$ subunits (14). CK2 is a pleiotropic kinase with more than 300 substrates (15). Consequently, CK2 is involved in multiple metabolic events. CK2 is found in the nucleus, cytoplasm, and organelles such as Golgi, ribosomes, and endoplasmic reticulum (16). Substantial evidence suggests that CK2 plays important roles in the regulation of cell proliferation, transformation, and apoptotic death $(14,17-20)$ as well as in pathological conditions (21). However, the mechanism by which CK2 regulates its activity and substrates remains largely unknown. It has been reported that CK2 plays a potential role in Nrf2 activation and degradation (22-24). However, to date, no studies have examined whether CK2 directly affects HO-1 gene expression. 
Herein, we report that $\mathrm{CK} 2$ regulates peroxynitrite-induced HO-1 expression in primary rat articular chondrocytes. We also demonstrate a possible role for the CK2 signaling pathway in the activation of $\mathrm{Nrf} 2$ to regulate $\mathrm{HO}-1$ expression.

\section{Materials and methods}

Reagents. 3-Morpholinosydnonimine hydrochloride (SIN-1), 5,6-dichlorobenzimidazole riboside (DRB), 4,5,6,7-tetrabromobenzotriazole (TBB), protease inhibitor cocktail, trypan blue $(0.4 \%)$, collagenase and paraformaldehyde were purchased from Sigma-Aldrich (St. Louis, MO, USA). Fetal bovine serum (FBS), Dulbecco's modified Eagle's medium (DMEM) and other culture reagents were purchased from Hyclone (Logan, UT, USA). Anti-HO-1, Nrf2, CK2 $\alpha$, and $\beta$-actin antibodies were obtained from Santa Cruz Biotechnology, Inc. (Santa Cruz, CA, USA). Anti-p-CK2 $\alpha$ antibody was purchased from Abcam (Cambridge, MA, USA). The horseradish peroxidase (HRP)-conjugated secondary antibody and the enhanced chemiluminescence (ECL) western blotting kit were obtained from Amersham Pharmacia Biotech (Piscataway, NJ, USA).

Cell culture of articular chondrocytes. Articular chondrocytes for primary culture were isolated from slices of knee-joint cartilage from 5-week-old female Sprague-Dawley rats (Samtako Co., Korea). Chondrocytes were isolated by enzymatic digestion for $1 \mathrm{~h}$ with $0.2 \%$ type II collagenase in DMEM. After collection of chondrocytes by brief centrifugation, cells were resuspended in DMEM supplemented with $10 \%$ heat-inactivated FBS and antibiotics $(50 \mathrm{U} / \mathrm{ml}$ of penicillin and $50 \mu \mathrm{g} / \mathrm{ml}$ of streptomycin) at $37^{\circ} \mathrm{C}$ in an atmosphere of $5 \% \mathrm{CO}_{2}$ and air. Cells were plated on culture dishes at a density of $5 \times 10^{4}$ cells $/ \mathrm{cm}^{2}$. The culture medium was replaced every 2 days, and cells reached confluence after 4-5 days in culture, designated as passage 0 (P0). P0 cells were then serially subcultured up to P6 by plating cells at a density of $5 \times 10^{4}$ cells $/ \mathrm{cm}^{2}$.

TUNEL assay for detection of apoptotic cells. Cells were washed with $1 \%$ phosphate-buffered saline (PBS)/BSA and fixed in $4 \%$ paraformaldehyde for $15 \mathrm{~min}$. Cells were then washed with PBS/BSA and permeabilized in $0.1 \%$ Triton X-100 for $5 \mathrm{~min}$ on ice. Next, fluorescein isothiocyanate (FITC)conjugated dUTP was used to label the cells with nicked DNA using the Apo-BrdU ${ }^{\mathrm{TM}}$ TUNEL assay kit (Invitrogen, Carlsbad, CA, USA) according to the manufacturer's instructions.

Western blot analysis. Equal amounts $(20 \mu \mathrm{g})$ of total proteins were loaded onto $12 \%$ SDS-polyacrylamide gels for electrophoresis. The proteins were then transferred to a nitrocellulose membrane using an electroblotting apparatus (Bio-Rad, Richmond, $\mathrm{CA}$ ), and the membranes were incubated with each primary antibody. Blots were incubated with an HRP-conjugated anti-rabbit secondary antibody. The membranes were developed with the ECL reaction system and visualized using the LAS-3000 Luminescent Image Analyzer (FujiFilm, Japan). Image Gauge Ver. 3.0 software was used to calculate differences in protein expression. $\beta$-actin was used as an internal control to monitor equal protein loading.
CK activity assay. CK2 activity was measured in cell lysates with a CK2 assay kit (CycLex Co., Japan) according to the manufacturer's recommended protocol. Kinase activity was calculated by subtracting the mean of the background control samples without enzyme from the mean of samples with enzyme.

Transfection of siRNA. A 21-nucleotide RNA duplex with 3'-dTdT overhangs was designed to interfere exclusively with rat CK $2 \alpha$ mRNA (Dharmacon, Lafayette, CO, USA) in the 'ready-to-use' option. A negative control siRNA was also provided by Dharmacon. CK2 $\alpha$ siRNA was introduced into chondrocytes using the DharmaFECT ${ }^{\circledast}$ DUO transfection reagent according to the manufacturer's instruction.

Immunofluorescence staining. Chondrocytes were cultured on collagen-coated 4-well glass chamber slides for $48 \mathrm{~h}$ in DMEM containing 10\% FBS. Cells were fixed in $4 \%$ paraformaldehyde/PBS followed by permeabilization in $0.2 \%$ Triton X-100/PBS. Appropriate primary antibodies were added to the cells for $30 \mathrm{~min}$ at $37^{\circ} \mathrm{C}$. For secondary labeling, cells were incubated with a FITC-conjugated secondary antibody (Invitrogen) for $30 \mathrm{~min}$ at $37^{\circ} \mathrm{C}$. Nuclei were counterstained using propidium iodide (PI). Fluorescent images were visualized and analyzed with a laser-scanning confocal microscope.

Immunoprecipitation. Cell lysates were incubated with protein A-sepharose beads at $4^{\circ} \mathrm{C}$ for $30 \mathrm{~min}$. Following incubation, beads and non-specific proteins were removed, and the cleared supernatant was incubated with a monoclonal antibody against CK2 $\alpha$. After incubation for $1 \mathrm{~h}$, immune complexes were captured by protein A-sepharose. Immunoprecipitates were washed with $1 \%$ Triton X-100/PBS several times, and proteins were then released by boiling in SDS sample buffer.

Statistics or reproducibility. Each experiment was repeated at least 3 times. Data are expressed as the means \pm standard error (SE) from each independent experiment. Data for the experimental and control groups were tested for statistical significance by a one-tailed Student's t-test. $\mathrm{P}<0.05$ denoted statistically significant differences.

\section{Results}

Low concentration of SIN-1 induces HO-1 expression without inducing apoptosis in primary rat chondrocytes. SIN-1 is a peroxynitrite generator and simultaneously produces $\mathrm{NO}$ and superoxide. At high concentrations, SIN-1 can induce apoptotic cell death in many cell types. Therefore, the present study aimed to elucidate the exact mechanism of SIN-1 on HO-1 upregulation. SIN-1 dose-dependently upregulated HO-1 expression in primary chondrocytes (Fig. 1A). However, SIN-1 exposure also significantly increased the number of TUNELpositive chondrocytes (Fig. 1B). Therefore, the present study used a low concentration of SIN-1 that did not cause apoptosis in order to induce expression of HO-1 in primary rat articular chondrocytes. SIN-1 $(200 \mu \mathrm{M})$ induced HO-1 expression with spontaneous downregulation in chondrocytes in a different manner than high concentrations of SIN-1 (Fig. 1C). SIN-1induced HO-1 expression was inhibited by the NO scavenger, 
A

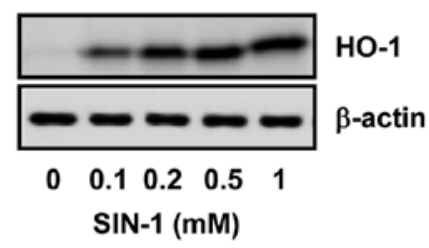

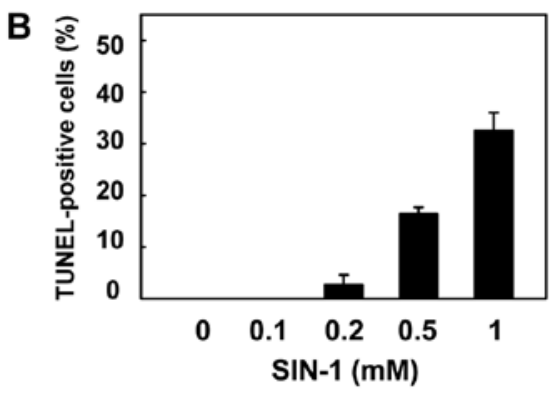

E
C

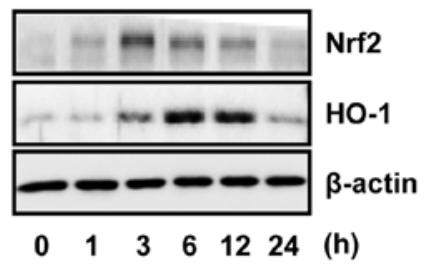

D

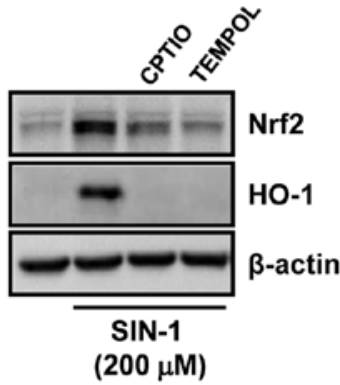

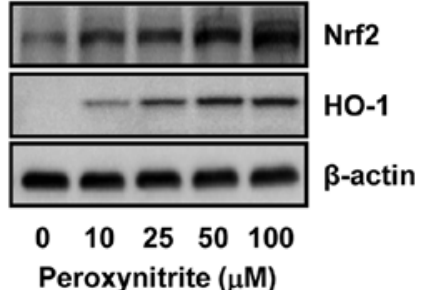

Figure 1. Effect of peroxynitrite on HO-1 expression in articular chondrocytes. (A) Primary rat chondrocytes were treated with various concentrations of SIN-1 for $12 \mathrm{~h}$. (B) After $24 \mathrm{~h}$ of SIN-1, DNA fragmentation was measured by the TUNEL assay. (C) Chondrocytes were treated with SIN-1 (200 $\mu \mathrm{M}$ ) for the indicated time periods. (D) Cells were treated with SIN-1 $(200 \mu \mathrm{M})$ for $6 \mathrm{~h}$ in the absence or presence of carboxy-PTIO (CPTIO) (250 $\mu \mathrm{M})$ or TEMPOL $(0.5 \mathrm{mM})$. (E) Chondrocytes were treated with various concentrations of peroxynitrite for $6 \mathrm{~h}$. Western blot analysis was performed with specific antibodies against Nrf2 and HO-1. $\beta$-actin was used as an internal control.

A

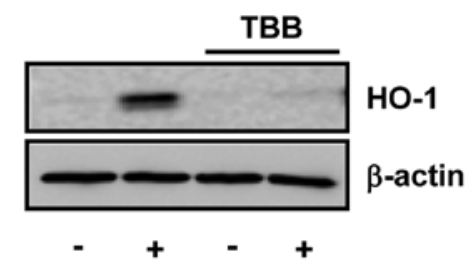

B

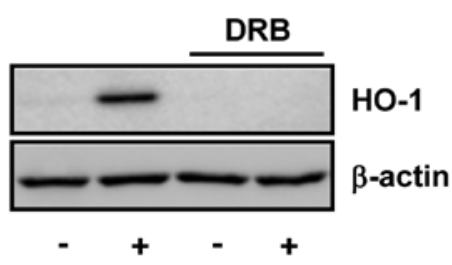

SIN-1

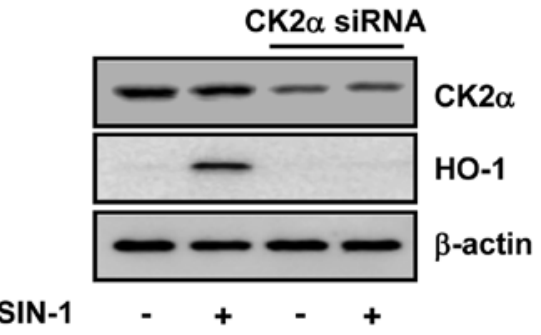

Figure 2. Involvement of CK2 in SIN-induced HO-1 expression in chondrocytes. (A) Cells were treated with SIN-1 $(200 \mu \mathrm{M})$ for $6 \mathrm{~h}$ in the absence or presence of TBB $(50 \mu \mathrm{M})$ or DRB $(50 \mu \mathrm{M})$. (B) After transfection with CK $2 \alpha$ siRNA, cells were treated with SIN-1 for $6 \mathrm{~h}$. Cell lysates were assessed by western blot analysis using $\mathrm{HO}-1$ and $\beta$-actin antibodies.

carboxy-PTIO and the superoxide dismutase (SOD) mimetic, TEMPOL in chondrocytes (Fig. 1D). This suggests that the effect of SIN-1 is caused by generation of peroxynitrite and that SIN-1 is a peroxynitrite donor. Finally, it was demonstrated that peroxynitrite induces the expression of Nrf2 and HO-1 in primary chondrocytes (Fig. 1E). These results indicate that the induction of HO-1 expression by SIN-1 was due to NO-mediated peroxynitrite rather than $\mathrm{NO}$ in primary rat chondrocytes.

Inhibition of CK2 activity prevents SIN-1-induced HO-I expression. To determine whether CK2 activity is associated with HO-1 expression in chondrocytes, the effects of CK2 inhibitors, TBB or DRB, on SIN-1-induced HO-1 expression were examined. Indeed, pretreatment with the CK2 inhibitor completely abolished SIN-1-induced HO-1 expression in chondrocytes (Fig. 2A). To confirm a specific effect of CK2 on SIN-1-induced HO-1 expression, CK2 was silenced using siRNA. Chondrocytes were transfected with a CK2 siRNA duplex or with a non-specific control for $24 \mathrm{~h}$. Cells were then incubated with SIN-1 or vehicle for another $6 \mathrm{~h}$. Transfection with the control siRNA did not significantly affect HO-1 protein levels relative to non-transfected controls (data not shown). As determined by western blot analysis, CK2 protein expression was markedly reduced in the CK2 siRNA-transfected chondrocytes. Similarly, siRNA efficiently silenced CK2 gene expression and completely suppressed CK2 levels (Fig. 2B). Importantly, knockdown of CK2 significantly decreased HO-1 expression induced by SIN-1. This suggests that CK2 activation is necessary for SIN-1-induced HO-1 expression in chondrocytes.

SIN-1 stimulates CK2 activity in chondrocytes. Next, the direct effect of SIN-1 on CK2 activity in chondrocytes was investigated using a CK2 activity assay. Because Nrf2 expression was detected $3 \mathrm{~h}$ after SIN-1 treatment (Fig. 1C), the CK2 
A

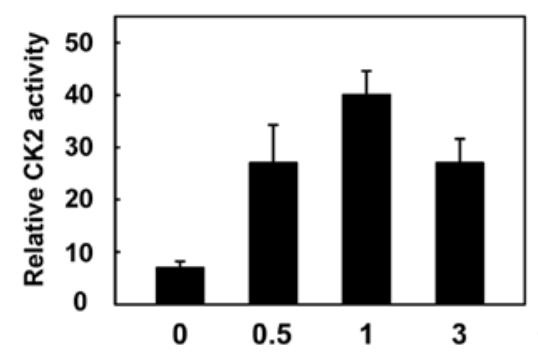

B

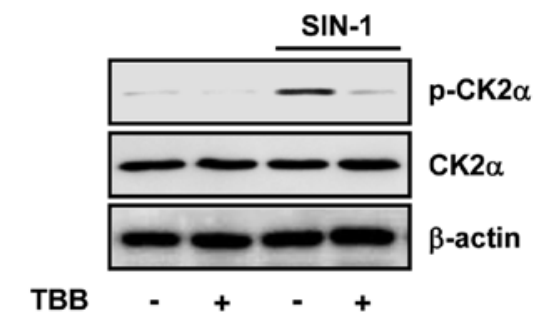

C

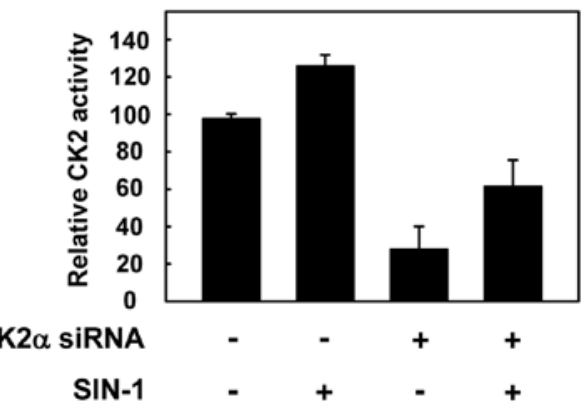

Figure 3. Effect of SIN-1 on the phosphorylation and activity of CK2. (A) Primary chondrocytes were treated with SIN-1 $(200 \mu \mathrm{M})$ for $0.5,1$ and $3 \mathrm{~h}$, and cell lysates were utilized in a CK2 activity assay. (B) Cells were treated with SIN $(200 \mu \mathrm{M})$ for $1 \mathrm{~h}$ in the absence or presence of TBB $(50 \mu \mathrm{M})$, and western blot analysis was performed using a specific p-CK2 $\alpha$ antibody. (C) After transfection with CK2 $\alpha$ siRNA, cells were treated with SIN-1 for $1 \mathrm{~h}$, and cell lysates were utilized in a CK2 activity assay.

activity assay was performed on lysates from chondrocytes treated with SIN-1 for $0.5,1$ and $3 \mathrm{~h}$. After SIN-1 treatment, CK2 activity increased, peaked after $1 \mathrm{~h}$, and then declined (Fig. 3A). In addition, SIN-1 significantly induced phosphorylation of CK2 $\alpha$, the catalytic subunit of the CK2 heterotetramer, in chondrocytes (Fig. 3B). TBB completely abrogated phosphorylation of CK2 by SIN-1. The effect of SIN-1 on CK2 activity was also determined using CK2 siRNA. Knockdown of CK2 significantly decreased CK2 activity induced by SIN-1 (Fig. 3C). This suggests that SIN-1directly stimulates the enzymatic activity of CK2 in chondrocytes.

CK2 induces HO-1 expression via Nrf2 activation in SIN-treated chondrocytes. To directly test whether CK2 interacts with Nrf2, a complementary set of immunoprecipitation and western blot analyses were performed. Chondrocytes were treated with SIN-1 for $1 \mathrm{~h}$, and the cells were immunoprecipitated with a CK2 $\alpha$ antibody and then detected with an antibody against Nrf2. Nrf2 was co-immunoprecipitated with CK2 $\alpha$ (Fig. 4A). Therefore, it was examined whether CK2 activation changes nuclear translocation of Nrf2 in chondrocytes stimulated with SIN-1. Cells were treated with SIN-1 for $3 \mathrm{~h}$ in the absence or presence of TBB. Then, cytoplasmic and nuclear extracts were analyzed by western blot analysis. The CK 2 inhibitor TBB completely abrogated SIN-1-induced nuclear translocation of Nrf2 to induce HO-1 expression (Fig. 4B). To this end, nuclear translocation of Nrf2 was determined by two-color analysis under confocal microscopy. In unstimulated cells, Nrf2 was detected in the cytoplasm (green fluorescence) but not in the nucleus, which was stained red by PI (Fig. 4C). At this time, CK2 expression was not changed by SIN-1 under these experimental conditions. However, when chondrocytes were incubated with SIN-1, there was a significant increase in nuclear translocation of Nrf2, which is in correlation with the cytoplasmic and nuclear distribution of Nrf2. TBB completely blocked the nuclear accumulation of Nrf2 induced by SIN-1. These results suggest that CK2 stimulates Nrf2 activation, which causes an increase in HO-1 expression in SIN-1-treated chondrocytes.

\section{Discussion}

HO- 1 is a tightly-regulated antioxidant enzyme that is induced as an adaptive mechanism against reactive oxygen species (ROS) in many cell types, including chondrocytes (25). Numerous studies have documented that NO is a potent inducer of HO-1 in various cell types, and the induction of HO-1 by NO donors occurs predominantly via a transcriptional mechanism (26-28). NO has a negligible role in mediating HO-1 expression induced by NO donors, such as sodium nitroprusside (SNP), which produces NO and iron $(29,30)$. In addition, it has been demonstrated that some NO donors can induce apoptotic cell death in many cell types (31-34). Further, the HO system has been reported to protect against the cytotoxic effects of high-concentration NO donors (35). Therefore, in the present study, we used a low concentration of SIN-1 to induce HO-1 expression without inducing apoptosis in primary rat chondrocytes.

On the other hand, NO generated through reacting with endogenous superoxide also leads to the formation of peroxynitrite, a short-lived and damaging oxidant that may have diverse negative effects on important biological molecules (36). Therefore, cells exposed to exogenous NO donors generate peroxynitrite because NO diffuses easily through membranes, and superoxide is constitutively produced in mitochondria under normal conditions. Furthermore, NO can counteract the actions of MnSOD and cytochrome oxidase, which increases superoxide production and peroxynitrite formation (37). Due to the multiple actions of NO donors, the exact mechanism underlying NO-induced $\mathrm{HO}-1$ expression has yet to be clarified.

$\mathrm{Nrf} 2$ is the main transcription factor activating the HO-1 gene. Under unstimulated conditions, Nrf2 is present in the cytosol complexed with Keap1, which is a negative modulator of Nrf2's nuclear translocation. Oxidative stressinduced modification of Keap1 results in Nrf2 release; $\mathrm{Nrf} 2$ then translocates into the nucleus. Many reports have demonstrated the roles of PI3K and mitogen-activated protein kinases (MAPKs) for nuclear translocation of Nrf2 $(12,13,38)$. However, this proposed mechanism remains controversial. Recently, CK2-mediated sequential phosphorylation was demonstrated to play a potential role in Nrf2 activation and degradation $(22,23)$. Therefore, the present study examined whether CK2 activity is directly associated 
A

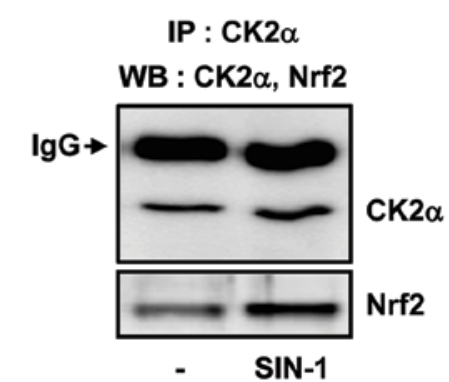

B

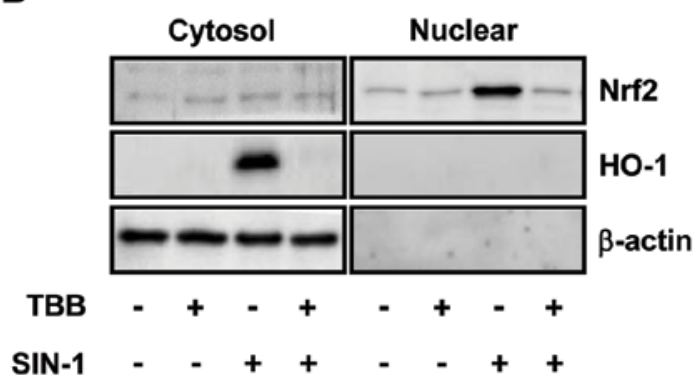

C

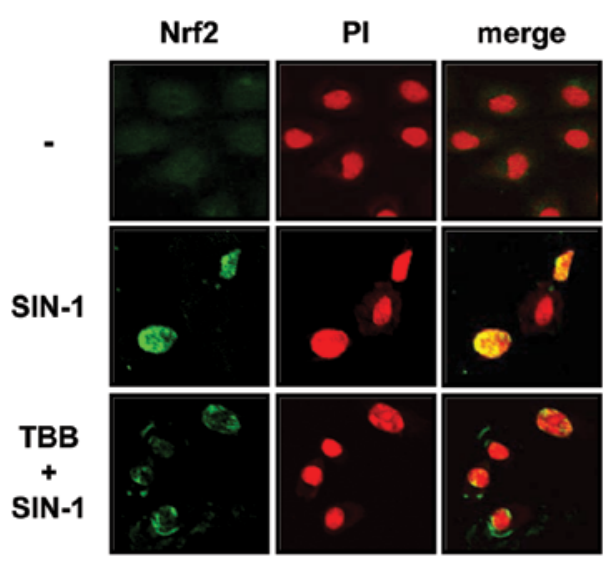

Figure 4. Regulation of Nrf2 activation by CK2. (A) Cells were treated with SIN-1 for 3 h and cell lysates were immunoprecipitated with a CK2 $\alpha$ antibody. Western blot analysis was performed with antibodies against CK2 $\alpha$ and Nrf2. (B) Cells were treated with SIN-1 (200 $\mu \mathrm{M}$ ) for $3 \mathrm{~h}$ in the absence or presence of TBB $(50 \mu \mathrm{M})$, and cytoplasmic and nuclear proteins were isolated and analyzed by western blot analysis. (C) Cells were treated with SIN-1 for $3 \mathrm{~h}$ in the absence or presence of TBB $(50 \mu \mathrm{M})$ and then analyzed by immunofluorescence using an Nrf 2 antibody. The right panels show superposition of Nrf 2 antibody and PI immunoreactivity (orange).

with SIN-1-induced Nrf2 activation and HO-1 expression in primary chondrocytes.

A low concentration of SIN-1 $(200 \mu \mathrm{M})$ induced HO-1 expression with spontaneous downregulation in primary rat chondrocytes. Further, our data showed that SIN-1-induced expression of HO-1 was inhibited by the NO scavenger, carboxyPTIO and the SOD mimetic, TEMPOL, in chondrocytes. We note that this is in contrast to a previous report showing that SIN-1-induced HO-1 expression was inhibited by an NO scavenger but not by SOD in arterial endothelial cells (39). Moreover, induction of HO-1 by SIN-1 was completely abrogated by the peroxynitrite scavenger, MnTBAP, in a concentration-dependent manner (data not shown). This suggests that induction of HO-1 by SIN-1 is due to peroxynitrite rather than NO in primary rat chondrocytes. Next, we investigated the involvement of the CK2 signaling pathway on SIN-1-induced expression of HO-1. Blockade of CK2 using a specific inhibitor or siRNA markedly decreased HO-1 expression induced by SIN-1 in chondrocytes. Additionally, SIN-1 treatment induced the phosphorylation and activity of CK2, which increased and peaked $1 \mathrm{~h}$ after treatment and declined thereafter. We also examined whether SIN-1 activates Nrf2 via CK2 signaling, which leads to induction of $\mathrm{HO}-1$ expression. Importantly, CK2 activation directly stimulated the phosphorylation and nuclear translocation of Nrf2 in chondrocytes stimulated with SIN-1.

Articular cartilage destruction is a key pathological characteristic of osteoarthritis. Recently, it has been reported that HO-1 induction by low doses of sodium nitroprusside can protect against apoptosis induced by high doses of SNP in human chondrocytes (40). These authors also suggested that inhibition of HO-1 activity significantly increased NO donorinduced cytotoxicity in chondrocytes. Thus, it is important to elucidate the regulation and function of HO-1 in articular diseases. Furthermore, this study raises the interesting possibility that HO-1 may be a potential therapy for diseases associated with apoptotic cell death. For this reason, it is important to identify the precise role of HO-1 in the maintenance and cell death of chondrocytes in articular cartilage.

\section{Acknowledgements}

This study was supported by the Korea Science and Engineering Foundation (grant no. R01-2007-000-20100-0).

\section{References}

1. Tenhunen R, Marver HS and Schmid R: The enzymatic conversion of heme to bilirubin by microsomal heme oxygenase. Proc Natl Acad Sci USA 61: 748-755, 1968.

2. Wilks A: Heme oxygenase: evolution, structure, and mechanism. Antioxid Redox Signal 4: 603-614, 2002.

3. Wu L and Wang R: Carbon monoxide: endogenous production, physiological functions, and pharmacological application. Pharmacol Rev 57: 585-630, 2005.

4. Otterbein LE and Choi AM: Heme oxygenase: colors of defense against cellular stress. Am J Physiol Lung Cell Mol Physiol 279: L1029-L1037, 2000.

5. Otterbein LE, Bach FH, Alam J, Soares M, Tao LH, Wysk M, Davis RJ, Flavell RA and Choi AM: Carbon monoxide has antiinflammatory effects involving the mitogen-activated protein kinase pathway. Nat Med 6: 422-428, 2000.

6. Duckers HJ, Boehm M, True AL, Yet SF, San H, Park JL, Clinton WR, Lee ME, Nabel GL and Nabel EG: Heme oxygenase-1 protects against vascular constriction and proliferation. Nat Med 7: 693-698, 2001.

7. Pae HO, Choi BM, Oh GS, Lee MS, Ryu DG, Rhew HY, Kim YM and Chung HT: Roles of heme oxygenase-1 in the antiproliferative and antiapoptotic effects of nitric oxide on Jurkat T cells. Mol Pharmacol 66: 122-128, 2004. 
8. Henrotin YE, Bruckner P and Pujol JP: The role of reactive oxygen species in homeostasis and degradation of cartilage. Osteoarthritis Cartilage 11: 747-755, 2003.

9. Guillen MI, Megias J, Gomar F and Alcaraz MJ: Heme oxygenase-1 regulates catabolic and anabolic processes in osteoarthritic chondrocytes. J Pathol 214: 515-522, 2008.

10. Ishii T, Itoh K, Takahashi S, Sato H, Yanagawa T, Katoh Y, Bannai S and Yamamoto M: Transcription factor Nrf2 coordinately regulates a group of oxidative stress-inducible genes in macrophages. J Biol Chem 275: 16023-16029, 2000.

11. Zhang DD and Hannink M: Distinct cysteine residues in Keap1 are required for Keap1-dependent ubiquitination of Nrf2 and for stabilization of Nrf2 by chemopreventive agents and oxidative stress. Mol Cell Biol 23: 8137-8151, 2003.

12. Kietzmann T, Samoylenko A and Immenschuh S: Transcriptional regulation of heme oxygenase-1 gene expression by MAP kinases of the JNK and p38 pathways in primary cultures of rat hepatocytes. J Biol Chem 278: 17927-17936, 2003.

13. Li MH, Cha YN and Surh YJ: Peroxynitrite induces HO-1 expression via PI3K/Akt-dependent activation of NF-E2-related factor 2 in PC12 cells. Free Radic Biol Med 41: 1079-1091, 2006.

14. Litchfield DW: Protein kinase CK2: structure, regulation and role in cellular decisions of life and death. Biochem J 369: 1-15, 2003.

15. Meggio F and Pinna LA: One-thousand-and-one substrates of protein kinase CK2? FASEB J 17: 349-368, 2003.

16. Faust $M$ and Montenarh M: Subcellular localization of protein kinase CK2. A key to its function? Cell Tissue Res 301: 329-340, 2000 .

17. Ahmed K, Gerber DA and Cochet C: Joining the cell survival squad: an emerging role for protein kinase CK2. Trends Cell Biol 12: 226-230, 2000.

18. Pinna LA: Protein kinase CK2: a challenge to canons. J Cell Sci 115: 3873-3878, 2002.

19. Ahmad KA, Wang G, Unger G, Slaton J and Ahmed K: Protein kinase CK2 - a key suppressor of apoptosis. Adv Enzyme Regul 48 : 179-187, 2008.

20. Issinger OG: Casein kinases: pleiotropic mediators of cellular regulation. Pharmacol Ther 59: 1-30, 1993.

21. Guerra B and Issinger OG: Protein kinase CK2 in human diseases Curr Med Chem 15: 1870-1886, 2008.

22. Pi J, Bai Y and Reece JM: Molecular mechanism of human Nrf2 activation and degradation: role of sequential phosphorylation by protein kinase CK2. Free Radic Biol Med 42: 1797-1806, 2007.

23. Apopa PL, He X and Ma Q: Phosphorylation of Nrf2 in the transcription activation domain by casein kinase 2 (CK2) is critical for the nuclear translocation and transcription activation function of Nrf2 in IMR-32 neuroblastoma cells. J Biochem Mol Toxicol 22: 63-76, 2008

24. Afonyushkin T, Oskolkova OV, Binder BR and Bochkov VN: Involvement of CK2 in activation of electrophilic genes in endothelial cells by oxidized phospholipids. J Lipid Res 52: 98-103, 2011.

25. Zwerina J, Tzima S, Hayer S, Redlich K, Hoffmann O, HanslikSchnabel B, Smolen JS, Kollias G and Schett G: Heme oxygenase 1 (HO-1) regulates osteoclastogenesis and bone resorption. FASEB J 19: 2011-2013, 2005 .
26. Bouton $\mathrm{C}$ and Demple B: Nitric oxide-inducible expression of heme oxygenase-1 in human cells: translation-independent stabilization of the mRNA and evidence for direct action of nitric oxide. J Biol Chem 275: 32688-32693, 2000.

27. Durante W, Kroll MH, Christodoulides N, Peyton KJ and Schafer AI: Nitric oxide induces heme oxygenase-1 gene expression and carbon monoxide production in vascular smooth muscle cells. Circ Res 80: 557-564, 1997.

28. Naughton P, Foresti R, Bains SK, Hoque M, Green CJ and Motterlini R: Induction of heme oxygenase 1 by nitrosative stress. J Biol Chem 277: 40666-40674, 2002.

29. Kurozumi R, Takahashi M and Kojima S: Involvement of mitochondrial peroxynitrite in nitric oxide-induced glutathione synthesis. Biol Pharm Bull 28: 779-785, 2005.

30. Kim HJ, Tsoy I, Park MK, Lee YS, Lee JH, Seo HG and Chang KC: Iron released by sodium nitroprusside contributes to heme oxygenase-1 induction via the cAMP-protein kinase A-mitogen-activated protein kinase pathway in RAW264.7 cells. Mol Pharmacol 69: 1633-1640, 2006.

31. Niknahad $\mathrm{H}$ and O'Brien PJ: Involvement of nitric oxide in nitroprusside-induced hepatocyte cytotoxicity. Biochem Pharmacol 51: 1031-1039, 1996.

32. Li MH, Cha YN and Surh YJ: Carbon monoxide protects PC12 cells from peroxynitrite-induced apoptotic death by preventing the depolarization of mitochondrial transmembrane potential. Biochem Biophys Res Commun 342: 984-990, 2006.

33. Lee SW, Song YS, Shin SH, Kim KT, Park YC, Park BS, Yun I, Kim K, Lee SY, Chung WT, et al: Cilostazol protects rat chondrocytes against nitric oxide-induced apoptosis in vitro and prevents cartilage destruction in a rat model of osteoarthritis. Arthritis Rheum 58: 790-800, 2008.

34. Nilakantan V, Liang H, Maenpaa CJ and Johnson CP: Differential patterns of peroxynitrite mediated apoptosis in proximal tubular epithelial cells following ATP depletion recovery. Apoptosis 13: 621-633, 2008.

35. Hara E, Takahashi K, Takeda K, Nakayama M, Yoshizawa M, Fujita H, Shirato K and Shibahara S: Induction of heme oxygenase-1 as a response in sensing the signals evoked by distinct nitric oxide donors. Biochem Pharmacol 58: 227-236, 1999.

36. Szabo C, Ischiropoulos $H$ and Radi R: Peroxynitrite: biochemistry, pathophysiology and development of therapeutics. Nat Rev Drug Discov 6: 662-680, 2007.

37. Poderoso JJ, Carreras MC, Lisdero C, Riobo N, Schopfer F and Boveris A: Nitric oxide inhibits electron transfer and increases superoxide radical production in rat heart mitochondria and submitochondrial particles. Arch Biochem Biophys 328: 85-92, 1996.

38. Yu R, Chen C, Mo YY, Hebbar V, Owuor ED, Tan TH and Kong AN: Activation of mitogen-activated protein kinase pathways induces antioxidant response element-mediated gene expression via a Nrf2-dependent mechanism. J Biol Chem 275: 39907-39913, 2000.

39. Polte T, Abate A, Dennery PA and Schröder H: Heme oxygenase-1 is a cGMP-inducible endothelial protein and mediates the cytoprotective action of nitric oxide. Arterioscler Thromb Vasc Biol 20: 1209-1215, 2000.

40. Kim HA, Lee KB and Bae SC: The mechanism of low-concentration sodium nitroprusside-mediated protection of chondrocyte death. Arthritis Res Ther 7: R526-R535, 2005. 